\title{
Fast variability of $\gamma$-ray emission from the supermassive black hole binary OJ 287
}

\section{levgen Vovk* and Andrii Neronov}

ISDC Data Center for Astrophysics, Geneva Observatory, Chemin d'Écogia 16, 1290 Versoix, Switzerland

E-mail: Ievgen.Vovk@unige.ch, Andrii.Neronov@unige.ch

\begin{abstract}
Here we report the discovery of the fast $\gamma$-ray variability of the blazar OJ 287. This blazar is known to host a binary supermassive black hole in its center. The observed variability occures on the time scale $T_{\text {var }} \lesssim 3-10$ hours, which is significantly shorter than the light crossing time of the primary black hole in the system. This indicates that the $\gamma$-ray emission is coming from the jet of the secondary, less massive black hole in the system $\left(1.3 \times 10^{8} M_{\odot}\right.$ vs. $\left.1.8 \times 10^{10} M_{\odot}\right)$. Detection of the $\gamma$-rays with energies higher than $10 \mathrm{GeV}$ allows us to conclude, that the Doppler factor of the jet is larger than 4. The study of the orbital modulation of emission of OJ 287 may make it be a unique laboratory for the study of the mechanism(s) of the formation of jets of black holes.
\end{abstract}

Fast X-ray timing and spectroscopy at extreme count rates: Science with the HTRS on the International $X$-ray Observatory - HTRS2011,

February 7-11, 2011

Champéry, Switzerland

* Speaker. 


\section{Introduction}

Although large-scale jets ejected by Active Galactic Nuclei (AGN) were discovered almost a century ago, origin of this phenomenon remains obscure up to present days (see [8] for a recent review). Blazar OJ 287 ( $z=0.306$ [16]) provides a unique laboratory for the study of the mechanism of AGN activity, because this is one of the few AGN known to host binary black hole system $[9,17]$. In this system, a lighter black hole of the mass $M_{\mathrm{BH} 1} \simeq 1.3 \times 10^{8} M_{\odot}$ orbits a heavier black hole of the mass $M_{\mathrm{BH} 2} \simeq 1.8 \times 10^{10} M_{\odot}$ with a period $P_{\text {orb }} \simeq 11.65 \mathrm{yr}[17,15]$. Separation of the components of the system at periastron is just about 10 Schwarzschild radii of the heavier black hole, so that the orbital motion is strongly affected by relativistic gravity effects [18].

Most of the existing studies of multi-wavelength blazar activity of OJ 287 adopt the assumption that observed jet is emitted by the larger black hole in the pair (see e.g. [17]). However, the emission in BL Lacs is strongly boosted by relativistic effects. Thus, if the less powerful jet emitted by the smaller black hole is aligned with the line of sight, while the jet from the larger black hole is not, the jet from the companion might dominate in the observed flux.

In what follows we show that variability properties of $\gamma$-ray emission from OJ 287 indicate that the relativistically beamed emission comes from the jet produced by the smaller black hole.

\section{Fermi observations}

In order to study variability of the $\gamma$-ray signal during the flaring activity, we have processed publicly available data of the LAT instrument over the period August 2008 - September 2010, using the Fermi Science Tools provided by the Fermi Science Support Centre. The data were selected using gtselect tool. The lightcurves were produced with the help of gtbin and gtexposure tools as it is explained in the Fermi data Analysis Threads ${ }^{1}$.

The lightcurve of the brightest flaring period, shown in Fig. 1, reveals several well separated flares. The brightest flare which happened on October 22, 2009 was reported by [5]. Follow

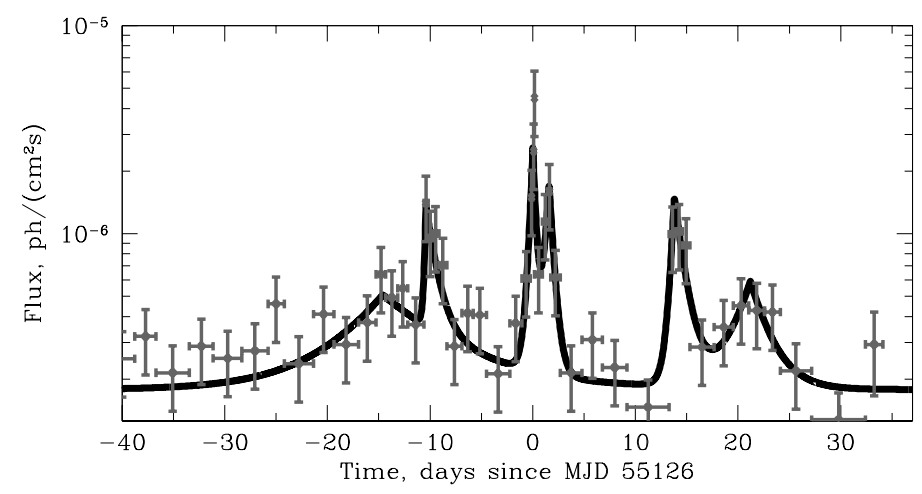

Figure 1: Lightcurve of October-November 2009 flare of OJ 287 in $E>0.1 \mathrm{GeV}$ energy band, binned in time bins with $\mathrm{S} / \mathrm{N}$ ratio equal to 3. Black curve shows model fit to the lightcurve, Eq. 2.1 with parameters given in Table 1.

\footnotetext{
${ }^{1}$ http://fermi.gsfc.nasa.gov/ssc/data/analysis/scitools/
} 


\begin{tabular}{c|cccc}
\hline $\mathrm{k}$ & $t_{k}$ & $t_{r k}$ & $t_{d k}$ & $\begin{array}{c}A_{k} \\
\mathrm{~d}\end{array}$ \\
& $\mathrm{~d}$ & $\mathrm{~d}$ & $\times 10^{-6} \mathrm{~cm}^{-2} s^{-1}$ \\
\hline 1 & $1.4 \pm 0.5$ & $5.0_{-2.5}^{+10.0}$ & $7.0_{-3.5}^{+4.0}$ & $0.33_{-0.08}^{+0.07}$ \\
2 & $5.6_{-0.3}^{+0.2}$ & $0.15_{-0.14}^{+0.37}$ & $1.0_{-0.4}^{+0.6}$ & $1.0_{-0.3}^{+0.4}$ \\
3 & $16.02_{+0.05}^{-0.05}$ & $0.33_{-0.09}^{+0.19}$ & $0.30_{-0.1}^{+0.15}$ & $2.3_{-0.6}^{+0.6}$ \\
4 & $17.6_{-0.15}^{+0.18}$ & $0.5_{-0.25}^{+0.4}$ & $0.46_{-0.18}^{+0.25}$ & $1.5_{-0.5}^{+0.5}$ \\
5 & $29.8_{-0.3}^{+0.4}$ & $0.4_{-0.23}^{+0.3}$ & $1.0_{-0.4}^{+0.5}$ & $1.3_{-0.3}^{-0.3}$ \\
6 & $37.2_{-1.0}^{+1.3}$ & $2.0_{-1.0}^{+1.8}$ & $1.9_{-2.2}^{-0.9}$ & $0.41_{-0.12}^{-0.14}$ \\
\hline
\end{tabular}

Table 1: Parameters of the model fit (see Eq. 2.1) to the lightcurve of the $\gamma$-ray flare of OJ 287 together with their $68 \%$ confidence ranges.

up observations of October 22 flare by Swift/XRT have revealed an increase of the X-ray flux accompanying the $\gamma$-ray flare [6].

OJ 287 is located close to the Ecliptic plane, and, in principle, the lightcurve can be affected by the Sun passing through the region of the sky close to the source. We have checked that this is not the case for the lightcurve in Fig. 1, as at the Sun was more than $15^{\circ}$ away from OJ 287 during the time interval in question.

Analysis of $\gamma$-ray images of the sky reveal few other sources in the vicinity of OJ 287. However, these sources are rather weak, so that they are not detected within the one-month exposure, corresponding to the duration of the flaring activity of OJ 287, and can not affect the results presented here.

The lightcurve of the flare consists of several well separated pulses with rather sharp rises and decays. To find the rise and decay times we have fitted the lightcurve with a phenomenological model of a sum of exponentially rising and decaying pulses

$$
F(t)=B+\sum_{k=1}^{3} \begin{cases}A_{k} \exp \left(\left(t-t_{k}\right) / t_{r k}\right), & t<t_{k} \\ A_{k} \exp \left(-\left(t-t_{k}\right) / t_{d k}\right), & t>t_{k}\end{cases}
$$

where $B=$ const is the background level. The background level was found from the circle of the radius $2^{\circ}$ displaced by 5 degrees from the source position. Parameters of the model function (2.1), derived from the fitting, are given in Table 1.

One can see from Fig. 1 and Table 1 that brightest flares are characterized by the rise/decay times of several hours. At this time scales measurement is complicated by the fact that Fermi/LAT telescope observes a given patch of the sky once in 3.2 hours (once per two rotation periods of $\simeq 96 \mathrm{~min}$ ). This can be seen from Fig. 2. Photons from the source and background regions come only within periodic time intervals spaced by $3.2 \mathrm{hr}$ marked by vertical grey strips in the two panels of the Fig. 2. It is clear that Fermi/LAT pointing pattern does not allow to constrain the rise/decay time of the flares to better than $3.2 \mathrm{hr}$. The apparently abrupt end of the flare limits the decay time of the flare to be less than $3.2 \mathrm{hr}$. Assuming that the source flux did not change in the time interval following the peak of the flare, one could estimate the chance probability of detecting zero photons in this time bin to be $7 \times 10^{-4}$. 


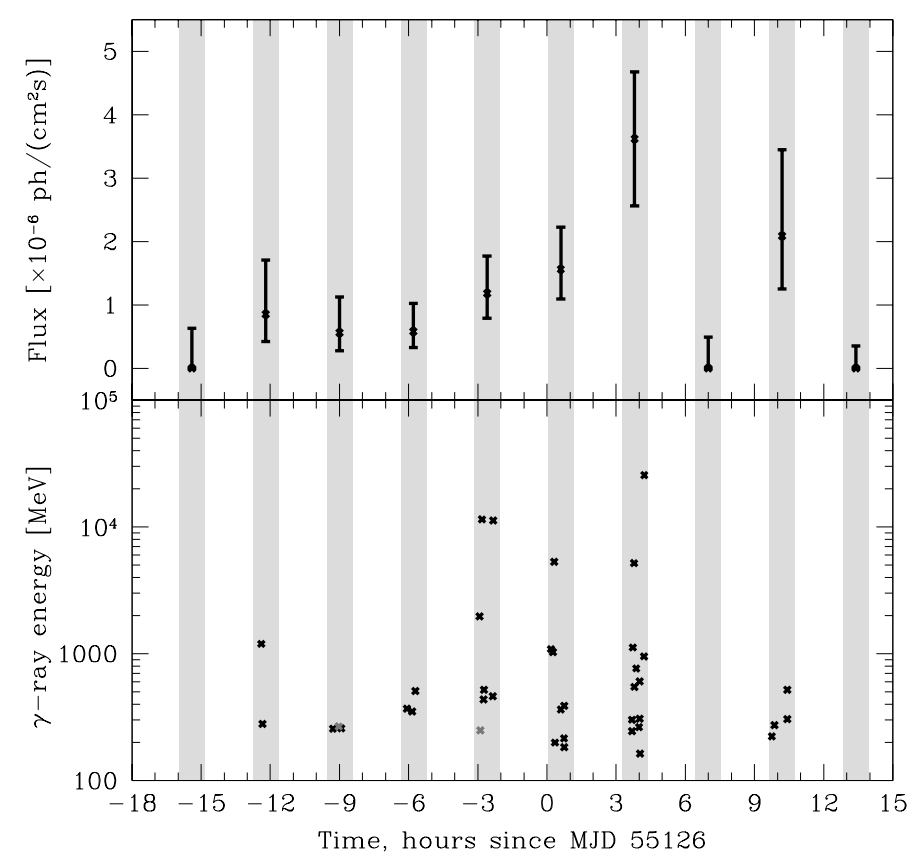

Figure 2: Upper panel: lightcurve of brightest episode of October-November 2009 flare of OJ 287 in $E>$ $0.1 \mathrm{GeV}$ energy band. Lower panel: energies and arrival times of $\gamma$ rays from the source (black points) and from the background region (grey points). Vertical grey strips show the periods when the source was in the field of view of the LAT telescope.

\section{Origin of the relativistic jet in $\mathrm{OJ} 287$}

Constraint on the variability time scale of the $\gamma$-ray flares $T_{\mathrm{var}}=\min \left(t_{r k}, t_{d k}\right) \leq 3.2 \mathrm{hr}$, derived above, enables us to identify the $\gamma$-ray emission site within the binary black hole system of OJ 287.

It is commonly accepted that the $\gamma$ ray emitting jets are generated by the AGN "central engines", the supermassive black holes, on the distance scales of the order of the gravitational radius $R_{g}$ of the supermassive black hole. Minimal variability time scale of the electromagnetic emission originating from the AGN central engine is expected to be not shorter than the light-crossing time of this black hole,

$$
\begin{aligned}
T_{\mathrm{lc}} & =2(1+z) R_{\mathrm{BH}} / c=2(1+z)\left(R_{g}+\sqrt{R_{g}^{2}-a^{2}}\right) / c \\
& \simeq\left\{\begin{array}{l}
0.5\left[M_{\mathrm{BH}} / 1.3 \times 10^{8} M_{\odot}\right] \mathrm{hr}, a=R_{g} \\
0.9\left[M_{\mathrm{BH}} / 1.3 \times 10^{8} M_{\odot}\right] \mathrm{hr}, a=0
\end{array}\right.
\end{aligned}
$$

where $R_{\mathrm{BH}}$ is the size of the black hole horizon and $0<a<R_{g}$ is the black hole's rotation momentum per unit mass. Variability at the time scale $T_{\mathrm{var}} \sim T_{\mathrm{lc}}$ is observed for a number of sources, powered by black holes $([1-3,7,10,11,13])$.

The light crossing time for the larger black hole in the system with $M_{\mathrm{BH}} \simeq 1.8 \times 10^{10} M_{\odot}$ varies from $120(a=0)$ to 70 hours $\left(a=M_{B H}\right)$, for the smaller one this transforms to 0.9 and 0.5 hours correspondingly. Thus the upper bound obtained here is much smaller than the light crossing time of the more massive black hole in the pair, but, at the same time, is larger than the light crossing time 
of the companion with the mass of $M_{\mathrm{BH}} \simeq 1.3 \times 10^{8} M_{\odot}$. Note, that this result does not depend on the assumptions about the relativistic motion of the $\gamma$-ray emission region, as the minimal possible time scale $\Delta T_{\min }$ of an emission from the relativistic jet moving with bulk Lorentz factor $\Gamma$ does not depend on $\Gamma$ and is instead determined by the size of the non-moving "central engine" which ejected the jet $[4,11,12]$. This implies that the observed $\gamma$-ray emission is generated by the jet emitted by the lower mass companion black hole in the system.

Such a conclusion may look counter-intuitive, unless one takes into account the relativistic beaming effects. $\gamma$-ray flux from the relativistically beamed jet, moving with bulk Lorentz factor $\Gamma$ at an angle $\theta$ with respect to the line of sight, is boosted by a factor $\delta^{4}$, where $\delta=(\Gamma(1-$ $\beta \cos \theta))^{-1}$ is the Doppler factor. A constraint on the bulk Lorentz factor of the jet produced by the $M_{\mathrm{BH}} \simeq 1.3 \times 10^{8} M_{\odot}$ black hole could be found under the assumption that the observed X-ray emission from the system [14] originates from the same jet region as the $\mathrm{GeV}$ emission.

The highest energy of $\gamma$-ray photons coming from the source is $E_{\gamma, \max } \geq 30 \mathrm{GeV}$ (see Fig. 2). $\gamma$ rays of such energies can produce $e^{+} e^{-}$pairs in the interactions with the X-ray photons of the energy

$$
E_{X} \geq \frac{\Gamma^{2} m_{e}^{2} c^{4}}{(1+z)^{2} E_{\gamma}} \simeq 0.5\left[\frac{\Gamma}{4}\right]^{2}\left[\frac{E_{\gamma, \max }}{30 \mathrm{GeV}}\right]^{-1} \mathrm{keV}
$$

where we have assumed that the typical collision angles for the photons emitted from the jet are $\alpha \simeq \Gamma^{-1}$. Apparent (relativistically beamed) luminosity of OJ 287 in the soft X-ray band is $L_{\text {app }} \simeq$ $3 \times 10^{44} \mathrm{erg} / \mathrm{s}$, which corresponds to the observed flux $F_{X} \simeq 10^{-12} \mathrm{erg} / \mathrm{cm}^{2} \mathrm{~s}$ [14]. Calculating the optical depth of the $\gamma$-ray emission region with the comoving size $R^{\prime} \simeq \delta c T_{\mathrm{var}} /(1+z)$ and luminosity $L^{\prime} \simeq \delta^{-4}(1+z)^{2} L_{X}$ one finds

$$
\tau_{\gamma \gamma} \simeq 0.5\left[\frac{\delta}{4}\right]^{-6}\left[\frac{L_{X}}{10^{44} \mathrm{erg} / \mathrm{s}}\right]\left[\frac{T_{\mathrm{var}}}{3.2 \mathrm{hr}}\right]^{-1}
$$

High energy $\gamma$ rays can escape from the source if $\tau_{\gamma \gamma}<1$. This condition imposes a restriction on the Doppler factor $\delta \gtrsim 4$.

Thus, the observed $\gamma$-ray flux from the jet is Doppler boosted by at least a factor $\delta^{4} \gtrsim 3 \times 10^{2}$. It is interesting to note that even if the emission from the higher mass black hole is not relativistically beamed towards the observer on the Earth, it might be noticed in the spectrum of the source. Indeed, assuming a simple Eddington-like scaling of the accretion rate and the jet luminosity with the black hole mass, $L_{i} \sim M_{\mathrm{BH} i}$, one finds that the relativistically beamed luminosity of the jet from the lighter black hole $L \sim \delta^{4} L_{1} \geq 10^{2} L_{1}$ could, in fact, be comparable to the overall luminosity of the heavier black hole, $L_{2} \sim\left(M_{\mathrm{BH} 2} / M_{\mathrm{BH} 1}\right) L_{1} \simeq 1.4 \times 10^{2} L_{1}$. Fast variability of the emission can, therefore, serve as a tool for identification of the contribution of the emission from the lighter black hole in the overall source spectrum.

\section{Conclusions}

To summarize, we find that the observations of OJ 287 in the $E>0.1 \mathrm{GeV}$ energy band constrain the minimal timescale of the flux variations of the source to be shorter than $3.2 \mathrm{hr}$. The upper limit on the minimal variability timescale imposes a restriction on the size of the jet formation region. We find that the size of the jet formation region in the OJ 287 system is much smaller than 
the size of the horizon of the more massive black hole in the pair. This means that the observed $\gamma$ ray emission is produced by the jet ejected from the smaller black hole. Higher apparent luminosity of the smaller mass companion is explained by the effect of the relativistic beaming of the $\gamma$-ray emission. Combining the X-ray and $\gamma$-ray data, we find a restriction on the Doppler factor of the $\gamma$ ray emitting part of the jet, $\delta \gtrsim 4$. The observed variability time scale indicates that the relativistic jet from the smaller black hole is formed close to the black hole horizon, well inside the last stable orbit around non-rotating supermassive black hole.

$\gamma$-ray data provide a new insight in the physical model of the binary supermassive black hole system in OJ 287. $\gamma$-ray flaring activity is produced in the connection with the passage of the smaller black hole through the accretion disk around the larger companion during the periods of the close approach of the two black holes in the periastron of the binary orbit. Interaction of the smaller companion with the larger mass black hole accretion disk leads to the transient episodes of the ejection into the relativistic jet from the smaller mass black hole. It appears that the transient jet from the small mass black hole happens to be aligned along the line of sight, the fact responsible for the BL Lac type appearance of the source. It is not clear a priori if the jet from the smaller mass black hole forms only during the periastron passage or it exists throughout the binary orbit. Systematic monitoring of the source evolution in $\gamma$-rays on the orbital (11.7 years) time scale, which is now possible with Fermi, might clarify this question.

\section{References}

[1] Aharonian F. et al., 2006, Science, 314, 1424.

[2] Aharonian F.A. et al., 2007, Ap.J., 664, L71.

[3] Albert J., et al. 2007, Ap.J., 669, 862.

[4] Celotti A.L., Fabian A., Rees M., 1998, MNRAS, 293, 239

[5] Ciprini S. et al., 2009, ATEL, 2256.

[6] D’Ammando F., et al., 2009, ATEL, 2267.

[7] Gaidos, J. A, 1996, Nature, 383, 319.

[8] Harris D. E.; Krawczynski H., ARA\&A, 2006, 44, 463.

[9] Lehto H.J., Valtonen M.J., 1996, Ap.J., 460, 207

[10] Neronov A. \& Aharonian F., 2007, Ap.J., 671, 85;

[11] Neronov A., Semikoz D., Sibiryakov S., 2008, MNRAS, 391, 949.

[12] Neronov A., Semikoz D., Sibiryakov S., 2008a, AIP Conference Proceedings, 1085, 545.

[13] Remillard R.A., McClintock J.E., 2006, ARA\&A, 44, 49.

[14] Seta H., et al., 2009, PASJ, 61, 1011.

[15] Sillanpää A., Haarala, S., Valtonen, M. J., Sundelius, B., Byrd, G. G., 1988, Ap.J., 325, 628.

[16] Stickel M., Fried, J. W., Kuehr, H., 1989, A\&AS, 80, 103.

[17] Valtonen M.J. et al., 2009, Ap.J., 698, 781.

[18] Valtonen M.J. et al., 2008, Nature, 452, 851. 